\section{Case Reports in Oncology}

\title{
Hydrogen Sulfide and Hydrogen Sulfide-Synthesizing Enzymes Are Altered in a Case of Oral Adenoid Cystic Carcinoma
}

\author{
Kim Dongsoo ${ }^{a} \quad$ Jie Chen ${ }^{b} \quad$ Eric Wei ${ }^{b}$ Junaid Ansaric ${ }^{c}$ Andrew Meram ${ }^{a}$ \\ Stavan Patel $^{\mathrm{a}} \quad$ Ghali Ghali $^{\mathrm{a}} \quad$ Christopher Kevil $^{\mathrm{b}}$ Rodney E. Shackelford ${ }^{\mathrm{b}}$ \\ aHead and Neck Oncologic/Microvascular Reconstructive Surgery, Department of Oral \\ and Maxillofacial/Head and Neck Surgery, Louisiana State University Health Sciences \\ Center, Shreveport, LA, USA; ${ }^{b}$ Department of Pathology, Louisiana State University Health \\ Shreveport, Shreveport, LA, USA; 'Department of Molecular and Cellular Physiology, \\ Louisiana State University Health Shreveport, Shreveport, LA, USA
}

\section{Keywords}

Hydrogen sulfide · Hydrogen sulfide-synthesizing enzymes · Oral adenoid cystic carcinoma

\begin{abstract}
Adenoid cystic carcinomas (ACC) constitute $1 \%$ of all head and neck malignancies and are very rare in the oral cavity. With $<60$ oral ACCs described, their pathobiology is incompletely understood. Here, we report a case of oral cavity ACC in a 54-year-old woman. Since recent studies have demonstrated that several human tumors overexpress the hydrogen sulfide $\left(\mathrm{H}_{2} \mathrm{~S}\right)$ synthesizing enzymes cystathionine- $\beta$-synthase (CBS), cystathionine $\gamma$-lyase (CSE), and 3-mercaptopyruvate sulfurtransferase (3-MST), and also show dysregulated $\mathrm{H}_{2} \mathrm{~S}$ levels, we examined these biomarkers in the oral ACC and compared the results to those of adjacent benign oral epithelium. Western blotting was used to compare the protein expression of CBS, CSE, 3-MST nicotinamide phosphoribosyl transferase, and mitoNEET in ACC and adjacent benign oral mucosae. High-performance liquid chromatography was used to quantify the differences in tissue $\mathrm{H}_{2} \mathrm{~S}$ concentrations between the two biopsy types. We found that all the proteins examined here were increased in the ACC compared to adjacent benign oral mucosae. Interestingly, $\mathrm{H}_{2} \mathrm{~S}$ concentrations were decreased approximately $30 \%$ in ACC compared to benign mucosae.
\end{abstract}


Thus, in one example of this rare tumor type, the enzymes that synthesize $\mathrm{H}_{2} \mathrm{~S}$ are increased, while tissue $\mathrm{H}_{2} \mathrm{~S}$ levels are lower than those found in adjacent benign oral mucosae. Although limited to a single rare tumor type, to our knowledge this is the second time $\mathrm{H}_{2} \mathrm{~S}$ concentrations have been directly quantified inside a human tumor. Last, our results may indicate that alterations in $\mathrm{H}_{2} \mathrm{~S}$ synthesis and metabolism may be important in the pathobiology of ACC.

(C) 2018 The Author(s)

Published by S. Karger AG, Basel

\section{Introduction}

Adenoid cystic carcinoma (ACC) is an uncommon tumor comprising about $1 \%$ of all head and neck tumors, with a yearly incidence rate of 3-4.5 cases per million [1, 2]. ACC was first described by Robin, Lorain, and Laboulbene in two articles published in 1853 and 1854, who identified the characteristic non-luminal basaloid cells with scant clear to eosinophilic cytoplasm and angulated hyperchromatic nuclei, often growing in a cribriform pattern. ACC has an indolent growth pattern with an aggressive long-term clinical pattern and a propensity for perineural invasion. ACC commonly occurs in the parotid, submandibular, and major salivary glands, accounting for $27.9 \%$ of all salivary gland malignancies [1, 2]. At the molecular level, ACCs often express c-kit, EGFR/HER-1, SOX4, SOX10, cyclin D1, and mutated p53, and show PI3K/AKT pathway activation and E-cadherin loss [1-3]. ACCs of the oral cavity are rare, with $<60$ cases described $[1,2,4]$. Histologically and immunohistochemically, oral ACCs resemble ACCs found at other anatomic locations, although few of these tumors have been examined in detail. Here, we described an oral ACC case and measured tumor and adjacent benign oral mucosae for hydrogen sulfide $\left(\mathrm{H}_{2} \mathrm{~S}\right)$ levels and $\mathrm{H}_{2} \mathrm{~S}$-synthesizing enzymes.

\section{Case Report}

A 54-year-old woman presented with a history of a swelling in the right posterior buccal mass, approximately $2.0 \mathrm{~cm}$ in size, that was accompanied by a throbbing pain. The patient was consented for surgery and, after an Institution Review Board approval, biopsies were taken for study. At surgery, a $1.5 \mathrm{~cm}$ area was marked circumferentially around the tumor with bovie electrocautery. Three 4-mm punch biopsies were obtained from the periphery of the margin, and three 4-mm punch biopsies of the central ACC tumor core were taken. These were immediately placed in marked Eppendorf tubes and placed in a liquid nitrogen bath. Less than $20 \mathrm{~s}$ passed between taking the punch biopsy and the biopsies being placed in liquid nitrogen. We use the term "benign oral mucosae" to describe the benign tissue punch biopsies. As the half-life of $\mathrm{H}_{2} \mathrm{~S}$ in tissues is approximately $2 \mathrm{~min}$, microdissecting the tissue was not possible if $\mathrm{H}_{2} \mathrm{~S}$ tissue concentrations were to be properly analyzed [5]. The samples were passed on to the Pathology Department for further analysis. Upon histopathologic analysis, a diagnosis of ACC was rendered, and the lesion was staged as pathologic stage pT4a pN0, stage group IVA. Representative H\&E sections of the tumor are shown in Figure 1.

To further analyze the ACC, we performed Western blotting on the ACC/benign mucosal tissue pair for cystathionine- $\beta$-synthase (CBS), cystathionine $\gamma$-lyase (CSE), 3-mercaptopyruvate sulfurtransferase (3-MST), nicotinamide phosphoribosyl transferase (Nampt), and mitoNEET. The Western blots were performed as previously described [6]. As shown in Figure 2 , the 3 enzymes that synthesize $\mathrm{H}_{2} \mathrm{~S}$ (CBS, CSE, and 3-MST, Fig. 2a-c) were increased in the ACC samples compared to the benign oral mucosa samples, with 3-MST being most highly 
induced. Additionally, Nampt and mitoNEET were also induced in the ACC compared to benign oral mucosa samples (Fig. 2d, e). Last, we measured bioavailable free $\mathrm{H}_{2} \mathrm{~S}$, acid-labile, and bound (sulfane sulfur) levels, as previously reported [7]. As shown in Figure 3, $\mathrm{H}_{2} \mathrm{~S}$ levels and the acid-labile (iron-bound) sulfur fractions were lower in the ACC case than was observed in the benign oral mucosae, while the sulfane fraction was increased in the tumor compared to the benign oral mucosae. To ensure accuracy of the sulfur pool measurements, each sulfur pool was analyzed three times, and the $p$ values were calculated. The free $\mathrm{H}_{2} \mathrm{~S}$ was significant at $p=0.0299$. The remaining sulfur pools did not show significant $p$ values.

\section{Discussion}

Here, we report a rare case of oral ACC. As there are few examples of this tumor type, we decided to analyze the $\mathrm{H}_{2} \mathrm{~S}$-synthesizing enzymes CBS, CSE, and 3-MST, along with Nampt and mitoNEET, and directly measured the free $\mathrm{H}_{2} \mathrm{~S}$ pool, and the acid-labile and bound (sulfane sulfur) cellular pools. $\mathrm{H}_{2} \mathrm{~S}$ is a recently discovered gasotransmitter that promotes increased cancer cell growth and cell cycle progression, metastasis, invasion, angiogenesis, and chemotherapy resistance $[5,7,8]$. As has been found in several other human tumors, we found the $\mathrm{H}_{2} \mathrm{~S}$-synthesizing enzymes were increased in the ACC compared to benign oral mucosa samples (Fig. 2a-c). While the relative activities of the $\mathrm{H}_{2} \mathrm{~S}$-syntheizing enzymes were not measured, the increases in CBS, CSE, and 3-MST suggest that increased $\mathrm{H}_{2} \mathrm{~S}$ synthesis is part of the ACC tumor biology. Nampt is increased in several human malignancies; and, interestingly, Nampt coregulates CBS and CSE, with inhibition of either enzyme lowering cellular Nampt levels, and Nampt inhibition lowering CBS and CSE protein expression $[6,9,10]$. Our finding that it is increased in this case of ACC is not surprising, as increased Nampt expression appears to be a common event in human malignancies.

MitoNEET was highly increased in the oral ACC compared to benign oral mucosae (Fig. 3e). MitoNEET is increased in several human tumors, and high mitoNEET expression suppresses apoptosis and autophagy and lowers intramitochondrial iron concentrations, likely allowing tumor cells to tolerate higher reactive oxygen species, while avoiding ferroptosis $[6$, 11]. Last, we found that free $\mathrm{H}_{2} \mathrm{~S}$ and the iron-bound sulfur pool were lower in the ACC compared to benign oral mucosae, while the bound or sulfane faction was increased in the ACC (Fig. 3). This finding is interestingly in light of the increased ACC expression of CBS, CSE, and 3-MST. Previously, we found that oral squamous cell carcinoma had elevated CBS, CSE, and 3MST protein expression compared to benign oral mucosae, while free $\mathrm{H}_{2} \mathrm{~S}$ was significantly but also only slightly increased, on average, in the malignant carcinoma.

We concluded that oral squamous cell carcinoma likely produces high amounts of $\mathrm{H}_{2} \mathrm{~S}$ and rapidly metabolizes it as part of tumor maintenance and growth [6]. Our findings here give some support to that hypothesis, as ACC shows increased $\mathrm{H}_{2} \mathrm{~S}$-synthesizing enzymes, but lower free $\mathrm{H}_{2} \mathrm{~S}$ tissue concentrations. The acid-labile/iron-bound sulfur fraction is largely mitochondrial $[5,7,8]$. Since increased mitoNEET, which we observed here, lowers mitochondrial iron concentrations, this finding may reflect ACC mitochondrial alterations secondary to increased mitoNEET levels, along with other factors [11]. Last, the increase in the ACC sulfane pool may reflect increased $\mathrm{H}_{2} \mathrm{~S}$ sulfur moving into this sulfur pool.

The obvious limitation of this study is that only a single very rare tumor type was examined. Our results suggest that dysregulated $\mathrm{H}_{2} \mathrm{~S}$ metabolism is part of oral ACC and likely ACC biology in general. Furthers studies are needed. 


\section{Statement of Ethics}

The authors have no ethical conflicts to disclose.

\section{Disclosure Statement}

The authors declare no conflict of interest.

\section{References}

1 Coca-Pelaz A, Rodrigo JP, Bradley PJ, Vander Poorten V, Triantafyllou A, Hunt JL, et al. Adenoid cystic carcinoma of the head and neck-an update. Oral Oncol. 2015 Jul;51(7):652-61.

2 Jaso J, Malhotra R. Adenoid cystic carcinoma. Arch Pathol Lab Med. 2011 Apr;135(4):511-5.

3 Liu J, Shao C, Tan ML, Mu D, Ferris RL, Ha PK. Molecular biology of adenoid cystic carcinoma. Head Neck. 2012 Nov;34(11):1665-77.

4 Terada T. Adenoid cystic carcinoma of the oral cavity: immunohistochemical study of four cases. Int J Clin Exp Pathol. 2013 Apr;6(5):932-8.

5 Vitvitsky V, Kabil O, Banerjee R. High turnover rates for hydrogen sulfide allow for rapid regulation of its tissue concentrations. Antioxid Redox Signal. 2012 Jul;17(1):22-31.

6 Meram AT, Chen J, Patel S, Kim DD, Shirley B, Covello P, et al. Hydrogen Sulfide is Increased in Oral Squamous Cell Carcinoma Compared to Adjacent Benign Oral Mucosae. Anticancer Res. 2018 Jul;38(7):3843-52.

7 Leskova A, Pardue S, Glawe JD, Kevil CG, Shen X. Role of thiosulfate in hydrogen sulfide-dependent redox signaling in endothelial cells. Am J Physiol Heart Circ Physiol. 2017 Aug;313(2):H256-64.

8 Wu D, Si W, Wang M, Lv S, Ji A, Li Y. Hydrogen sulfide in cancer: friend or foe? Nitric Oxide. 2015 Aug;50:3845.

9 Shackelford RE, Mayhall K, Maxwell NM, Kandil E, Coppola D. Nicotinamide phosphoribosyltransferase in malignancy: a review. Genes Cancer. 2013 Nov;4(11-12):447-56.

10 Sanokawa-Akakura R, Ostrakhovitch EA, Akakura S, Goodwin S, Tabibzadeh S. A H2S-Nampt dependent energetic circuit is critical to survival and cytoprotection from damage in cancer cells. PLoS One. 2014 Sep;23:e108537.

11 Mittler R, Darash-Yahana M, Sohn YS, Bai F, Song L, Cabantchik IZ, et al: NEET proteins: A new link between iron metabolism, reactive oxygen species, and cancer. Antioxid Redox Signal. 2018 Mar. doi:10.1089/ars.2018.7502. 


\section{Case Reports in Oncology}

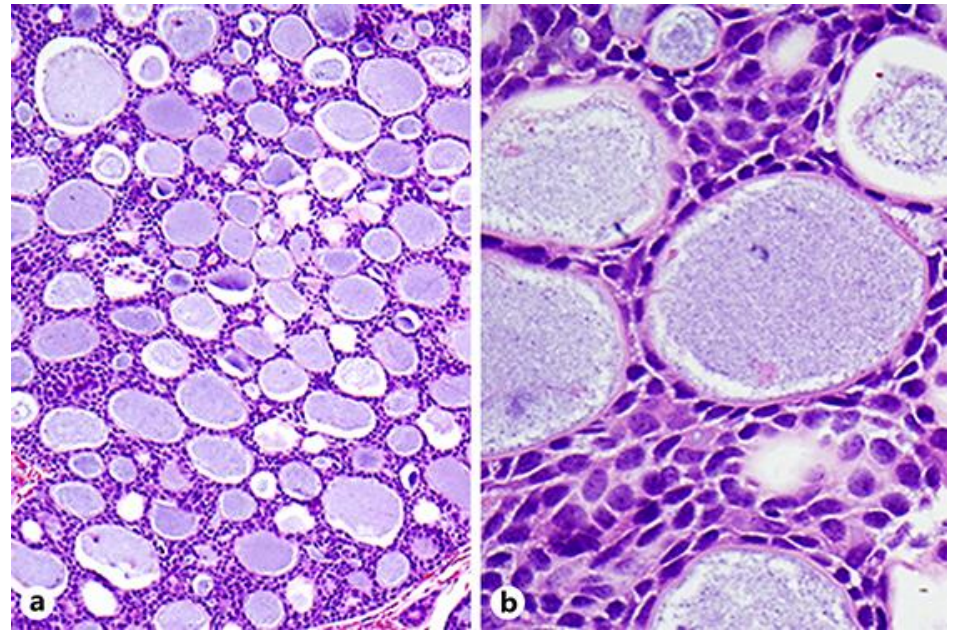

Fig. 1. Low-power (a) and high-power (b) images of the adenoid cystic carcinoma tumor by H\&E staining.
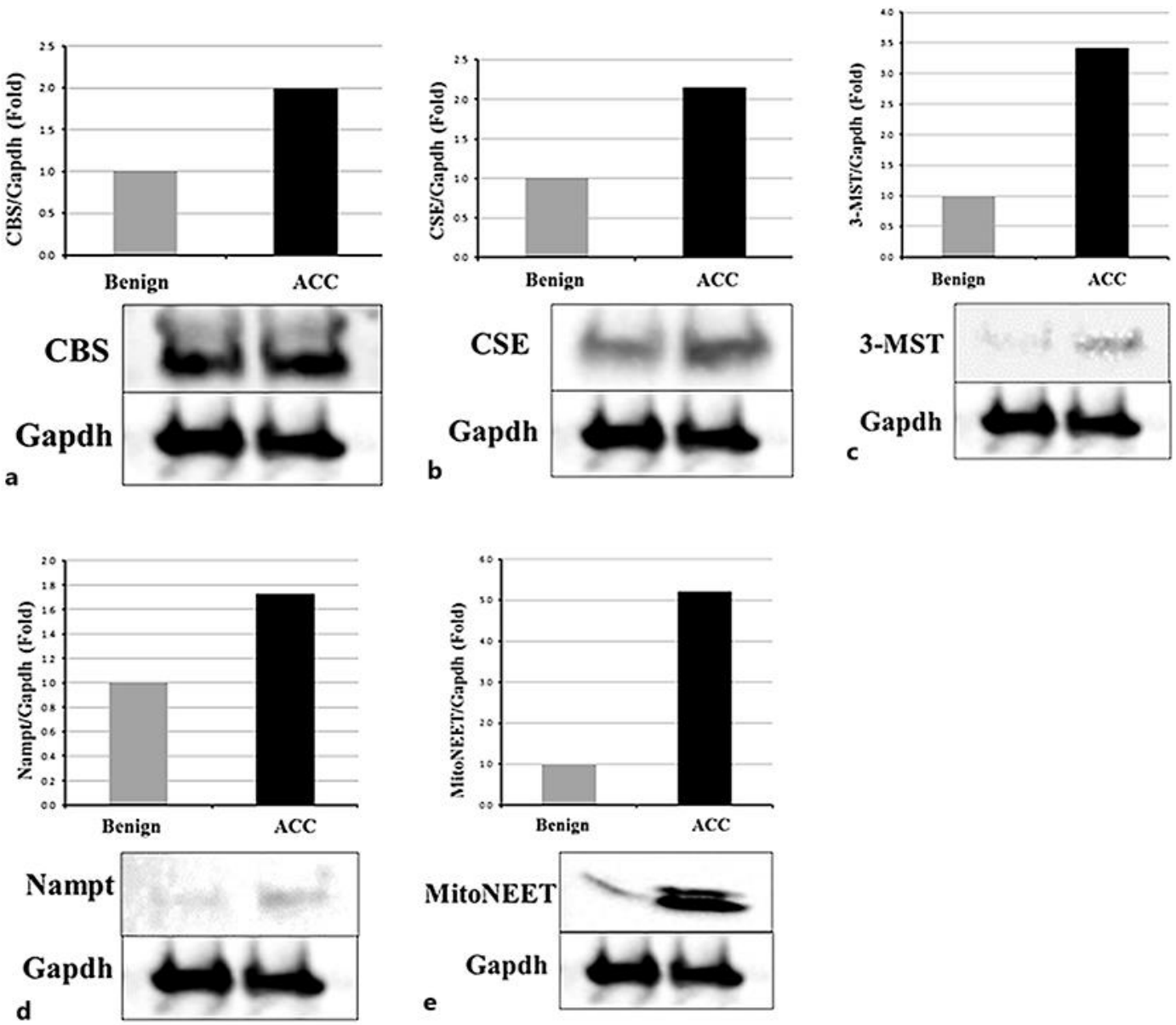

Fig. 2. Western blot analyses of benign oral mucosae and the oral adenoid cystic carcinoma (ACC) for cystathionine- $\beta$-synthase (CBS, a), cystathionine $\gamma$-lyase (CSE, b) 3-mercaptopyruvate sulfurtransferase (3MST, c), nicotinamide phosphoribosyl transferase (Nampt, d), and mitoNEET (e). 


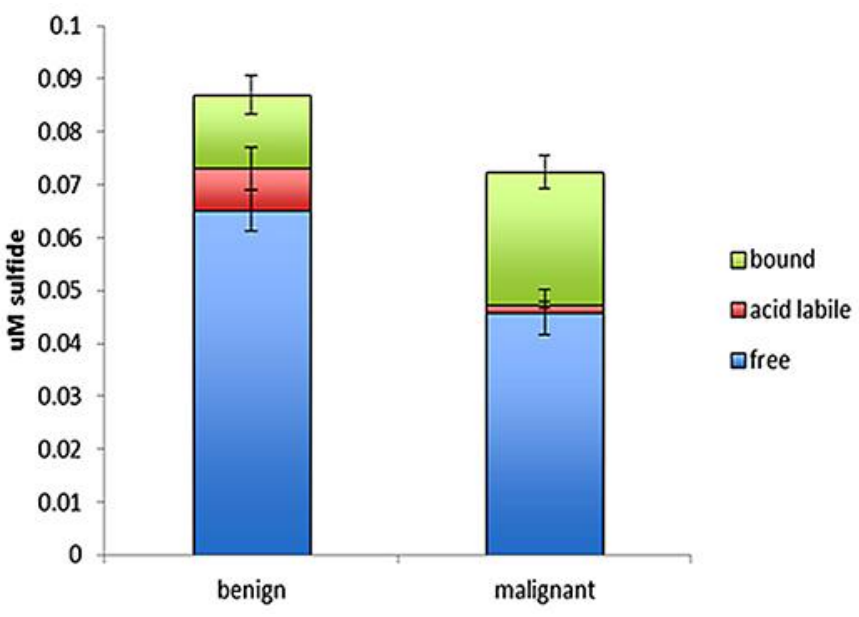

Fig. 3. Comparison of the cellular $\mathrm{H}_{2} \mathrm{~S}$ pools of benign oral mucosae and the oral adenoid cystic carcinoma case. Free $\mathrm{H}_{2} \mathrm{~S}$ pool (blue), the acid-labile fraction ("iron-bound" faction, red), and the bound (sulfane sulfur) pool (green). 